\title{
Mondoux André, Ménard Marc : Big Data et société. Industrialisation des médiations symboliques
}

Joachim Schöpfel

\section{(2) OpenEdition}

\section{Journals}

Édition électronique

URL : http://journals.openedition.org/edc/8990

DOI : $10.4000 /$ edc.8990

ISSN : 2101-0366

Éditeur

Université de Lille

Édition imprimée

Date de publication : 1 juin 2019

Pagination : 225-229

ISBN : 978-2-917562-21-5

ISSN : $1270-6841$

Référence électronique

Joachim Schöpfel, « Mondoux André, Ménard Marc : Big Data et société. Industrialisation des

médiations symboliques », Études de communication [En ligne], 52 | 2019, mis en ligne le 01 juin 2019, consulté le 21 janvier 2021. URL : http://journals.openedition.org/edc/8990 ; DOI : https://doi.org/ $10.4000 /$ edc. 8990

Ce document a été généré automatiquement le 21 janvier 2021.

(c) Tous droits réservés 


\title{
Mondoux André, Ménard Marc : Big Data et société. Industrialisation des médiations symboliques
}

\author{
Joachim Schöpfel
}

\section{RÉFÉRENCE}

Mondoux A., Ménard M. (dir.) (2018). Big Data et société. Industrialisation des médiations symboliques, Québec, Presses de l'Université du Québec.

1 Le premier livre du Groupe de recherche sur l'information et la surveillance au quotidien (GRISQ ${ }^{1}$ ) se distingue du flot de livres sur le «Big Data» par son approche multidisciplinaire, dialectique et critique. Souvent, les auteurs du «Big Data » font du marketing technologique ou versent dans l'apologie des mégadonnées ou données massives, quand ils n'expliquent pas simplement "comment faire avec", côté consommateur ou dans les entreprises et administrations. Ici, rien de cela.

2 Lancé en 2011 avec un financement du Conseil de recherches en sciences humaines du Canada, le GRISQ est composé d'une jeune équipe de chercheurs et étudiants spécialisés en sociologie, communication, sémiologie, économie et politique, pour développer une approche multidisciplinaire des rapports entre société, technologie, média et surveillance. Le GRISQ est également porteur de l'axe 2 «Big Data et surveillance » du Centre de recherche interuniversitaire sur la communication, l'information et la société (CRICIS) de l'Université du Québec à Montréal (UQAM). ${ }^{2}$ Au fil des ans, le GRISQ a ainsi mis en œuvre plusieurs projets de recherche autour de différentes thématiques, dont l'intégration de la géolocalisation dans les médias socionumériques, le phénomène du Big Data et l'application des rapports et modalités propres aux jeux vidéo à d'autres contextes communicationnels (ludification) comme stratégie de collecte de données.

L'origine du projet «Big Data, gouvernance et surveillance » remonte à 2013, avec un financement de l'UQAM pour mieux cerner «comment le Big Data s'inscrit dans le 
contexte plus large de transformations des modes de communication et régulation sociale, plus spécifiquement selon une forme de gouvernance qui s'appuie sur des dispositifs en ligne [...] comprendre comment le Big Data est lié à la fois à des modalités de représentations et de surveillance (et) révéler les fondements idéologiques du Big Data et ses effets sur la communication ».

L'ouvrage « Big Data et société » constitue une première synthèse collective de tous ces travaux. Mais synthèse n'est peut-être pas le mot qui convient, trop définitif, trop systématique pour une publication qui se veut avant tout l'expression d'un "effort collectif » d'une nouvelle génération de chercheuses et chercheurs dont le GRISQ « fut l'heureux témoin de passage ». Plus de la moitié des auteurs sont des doctorants, avec des contributions originales et neuves, et il faut sans doute lire l'ouvrage ainsi : non pas comme un résultat ou l'aboutissement d'un projet, mais comme un forum scientifique, comme une réflexion dynamique, un débat en construction, à partir d'une approche critique et dialectique qui interroge le Big Data «comme une phase de dialectique d'individuation sociétale : oui, le Big Data est producteur d'impacts, certains favorables, mais il est également produit par des dynamiques sociales qui font tout aussi partie de sa genèse ». L'ambition est double: ne pas évacuer sur le plan de l'épistémologie la dimension sociale ; et ne pas occulter sur le plan politique le rôle de l'idéologie comme dynamique normative. Un livre scientifique donc, certes, mais aussi un livre engagé, politique.

5 "Big Data et société« contient treize chapitres, rédigés

par douze chercheurs du GRISQ et deux auteurs invités,

Jodi Dean, professeur de sciences politiquesà

Hobart and William Smith Colleges (NY), et Mark Andrejevic, professeur de communication et des média

àMonash University (Australie). Les chapitres sont repartis en trois parties :

6 Économie politique du Big Data : en cinq chapitres, la première partie aborde les Big Data sous l'aspect de la "gouvernementalité dite 'algorithmique'", avec des études sur l'intelligence artificielle, le marché de l'emploi, l'exercice du pouvoir, les circuits marchands et les aspects juridiques. Jodi Dean introduit cette partie par une analyse marxiste du « capitalisme communicationnel (où) les valeurs démocratiques centrales sont concrétisées à travers les réseaux technologiques de communication». Un chapitre est consacré à l'usager (consommateur) comme sujet et à l'industrialisation du traitement de ses données personnelles (Marc Ménard et André Mondoux); un autre chapitre (Joëlle Gélinas) étudie le nouveau concept de la ville intelligente sous l'aspect du Big Data, de la production d'information et de la politique d'ouverture des données publiques (open data). Anne-Sophie Letellier analyse le concept du "data haven", refuge de données (zone refuge, paradis de données), un espace sécurisé et nonréglementé pour accéder à des données de façon permanente, en posant la question de la régulation étatique de l'information et en confrontant liberté d'expression, vie privée, droits de communication, intervention étatique, souveraineté territoriale et potentiel technologique.

7 Big Data et production de sens: les quatre chapitres de la deuxième partie sont axés sur la sémiotique. Le lecteur y trouvera aussi bien une critique de l'Internet des objets (André Mondoux) qu'une étude pointue de la théorie des données comme biens communs ("data commons", par Lisiane Lomazzi) et une approche globale des pratiques et usages des données massives (Maude Bonenfant et al.). 
8 Big Data, assujettissement et représentations culturelles: la dernière partie réunit quatre chapitres, dont le point commun est l'accent mis sur la subjectivité, le rôle de l'individu et du subjectif dans la production et la consommation des données massives. Mark Andrejevic pose la question du cadre de référence (ou plutôt de l'absence d'un tel cadre) dans l'usage des plateformes. Sans cadre médiatique, tout est vrai, tout est possible, tout se vaut ; « la logique du sans-cadre anticipe donc l'effondrement du sujet: à travers sa préemption d'un bord et son surpassement de l'autre. Traiter la totalité des informations possibles nécessite une perspective dépourvue de cadre, d'une vue de nulle part [...]». Les autres chapitres sont consacrés à la santé publique et la quantification du corps pour optimiser son rendement (Myriam Lavoie-Moore), à l'économie de l'attention (Marc Ménard et au cinéma documentaire (Diane Poitras).

9 La conclusion fait le lien entre ces études et l'actualité du Big Data, notamment le scandale Facebook/Cambridge Analytica et l'impact sur l'opinion publique (Brexit, élection de Donald Trump), les fausses nouvelles, l'ingérence étrangère dans les processus électoraux etc. Comment faire face à cette situation? Comment appréhender le Big Data dans la complexité des sociétés modernes? La conclusion mobilise les termes d'émancipation et de résistance, qui passent par une analyse audacieuse et nouvelle. Le lecteur qui ne partage pas cet enthousiasme citoyen ou l'approche critique, trouvera quand-même un regard jeune, un bol d'air frais et stimulant pour alimenter sa propre réflexion et ses propres travaux de recherche. Un regard neuf, pas naif. L'effort collectif dont le livre présente l'état actuel est le fruit de plusieurs années de recherche sur fond d'appropriation critique du phénomène de "Big Data" par les sciences humaines et sociales.

10 Le projet n'est pas terminé, car le GRISQ continue l'analyse des processus et stratégies de collecte massive de données. Cofinancés par la Fondation canadienne pour l'innovation (FCI), les travaux de l'équipe du GRISQ portent actuellement sur l'installation d'infrastructures de recherche permettant d'observer et d'analyser l'utilisation des médias socionumériques et les effets de la production massive de données personnelles sur la société, sur les applications utilisées, le temps de connexion et les données échangées (mobilité et géolocalisation), ou sur l'incitation à des temps de connexion plus longs vers des liens avec les jeux vidéo (ludification).

11 Le livre s'adresse aux étudiants et chercheurs en communication mais trouvera des lecteurs bien au-delà, en particulier (mais pas seulement) en sociologie et en économie. Le lecteur attend avec intérêt d'autres résultats des travaux de cette "nouvelle génération ". Une suite est déjà annoncée : le prochain numéro thématique de la revue tic\&société coordonné par André Mondoux, Marc Ménard et Maxime Ouellet sera consacré aux conséquences des dispositifs d'automatisation de la production, de la circulation et de la consommation des données massives sur les modalités de reproduction sociétale ; il paraitra en $2020^{3}$. 


\section{NOTES}

1. http://grisq.org/

2. https://www.cricis.uqam.ca/recherches/

3. https://journals.openedition.org/ticetsociete/2989

\section{AUTEURS}

\section{JOACHIM SCHÖPFEL}

Univ. Lille, EA 4073 - GERiiCO -

Groupement d'Études et de Recherche

Interdisciplinaire en Information

et Communication, F-59000 Lille, France

Joachim.schopfel@univ-lille.fr 